\title{
Correlation Denormalization in Interferometric or Polarimetric Radiometers: A Unified Approach
}

\author{
Andreas Colliander, Senior Member, IEEE, Francesc Torres, Senior Member, IEEE, and \\ Ignasi Corbella, Senior Member, IEEE
}

\begin{abstract}
This paper presents a general analysis of correlation measurements in an interferometer or a radiometer based on noise injection and/or switching and measurement of normalized correlations (e.g., imaging synthetic aperture or polarimetric radiometers). A compact unifying notation for denormalizing the measured normalized correlations in the presence of noise injection in one or both of the receiving channels is presented. Technological limitations are also assessed by evaluating the effect of associated approximations. Finally, the approach is validated by experimental results of the measurement and calibration of related front-end nonidealities, namely, the finite isolation of the front-end switch. The methods presented in this paper are illustrated by a thorough analysis of the so-called mixed baselines of microwave imaging radiometer using aperture synthesis, which refer to those baselines which are formed between the regular receivers (light-weight cost-efficient front-end) and the reference radiometers. These baselines require special attention, since the reference radiometers are noise-injection radiometers, which inject noise to the measured signal, whereas the regular receivers are total power receivers.
\end{abstract}

Index Terms-Denormalization, interferometric radiometry, Microwave Imaging Radiometer using Aperture Synthesis (MIRAS), polarimetric radiometer, synthetic aperture radiometry.

\section{INTRODUCTION}

A NY CORRELATING radiometer may be implemented with a correlator, which determines the normalized correlation between the signals received by two receivers [1]. This is also the case with the radiometers using interferometric aperture synthesis, which is a practical way to introduce imaging with high spatial resolution to radiometric measurements without introducing respective large real mechanically scanning

Manuscript received December 5, 2007; revised April 11, 2008 and June 10, 2008. First published November 25, 2008; current version published January 28, 2009. This work was supported in part by the European Space Agency as part of Noise Injection Radiometer Subsystem Project, which is part of the SMOS Payload Module Project Phase C/D, by Ulla Tuominen, by Jenny and Antti Wihuri, by Walter Ahlström, by the Finnish Cultural Foundations, and by the Spanish Research and EU Feder Project TEC2005-06863-C02-01/TCM.

A. Colliander was with the Helsinki University of Technology, 02150 Espoo, Finland, and the European Space Research and Technology Centre, European Space Agency, 2200 Noordwijk, The Netherlands. He is now with the Jet Propulsion Laboratory, California Institute of Technology, Pasadena, CA 91109 USA (e-mail: andreas.colliander@jpl.nasa.gov).

F. Torres and I. Corbella are with the Remote Sensing Laboratory, CRAE/IEEC-UPC, Universitat Politecnica de Catalunya, 08034 Barcelona, Spain, and also with the SMOS Barcelona Expert Centre on Radiometric Calibration and Ocean Salinity, 08003 Barcelona, Spain (e-mail: xtorres@ tsc.upc.edu; corbella@tsc.upc.edu).

Color versions of one or more of the figures in this paper are available at http://ieeexplore.ieee.org.

Digital Object Identifier 10.1109/TGRS.2008.2003247 antenna aperture [2]. This technique has already been demonstrated with a number of instruments, e.g., [3]-[6]. The cost of implementing an interferometric instrument comes in the form of system and calibration complexity [2], [7]. After the normalized correlations are obtained for each receiver pair, the total noise in the system needs to be accounted for in order to retrieve the visibility incident to the receivers; this process is called denormalization (e.g., [8]). For example, in [1] and [8], the denormalization is given in the case of total power radiometers. The purpose of this paper is to analyze also the case of noiseinjection radiometers (NIRs) and to provide a unified approach for all cases.

Microwave Imaging Radiometer using Aperture Synthesis (MIRAS) [9], [10] is the payload of European Space Agency's (ESA) Soil Moisture and Ocean Salinity (SMOS) mission [11], [12]. As the name reveals, the SMOS mission aims toward global measurement of soil moisture (e.g., [13], [14]) and ocean salinity (e.g., [15], [16]) at L-band, or $1.4 \mathrm{GHz}$. The MIRAS instrument is an interferometric aperture synthesis radiometer [17]-[19] which uses pairwise correlations of a thinned array of 69 receiving elements to form the measurement aperture. In order to obtain the normalized phase-calibrated correlation coefficient of each pairwise correlation, or baseline, the measurement results of MIRAS go through a series of corrections and calibrations [20]. The normalized correlations are then denormalized [21], [22] in order to account for the noise in the channels and retrieve the visibility of each baseline.

In MIRAS, the receiving elements also include three reference radiometers (NIR) [23], [24] to measure the average antenna temperature, or zero-baseline visibility, and the two noise levels of the calibration network [25]. These reference radiometers can also be used to form baselines with the regular receiver elements (Light-weight Cost-efficient Front-end as LICEF), which are called mixed baselines. However, in this case, the denormalization needs to take certain properties of the reference radiometer into account.

In [22] and [23], the denormalization principles related to the visibilities of MIRAS were presented. In this paper, a compact unifying formulation for the denormalization of all visibilities, including regular, mixed, and polarimetric zero baseline, is presented, which is applicable to all imaging synthetic aperture or polarimetric radiometers. In addition, the practical limitations of the linearization approximation (which significantly simplifies the processing) of the relation between the digital and analog correlations of mixed baselines is evaluated in the case of 1-bit correlator. Furthermore, it is shown that, in the case of mixed baselines, the finite isolation of the front-end switch 
needs to be taken into account, and experimental results on the calibration of this effect are presented.

First, Section II presents the theory of the denormalization and then the principles for taking the switching, noise injection, and finite isolation into account. Section III then unifies the denormalization equations with single coefficient. Finally, Section IV discusses in detail the effects of the linearization and isolation along with simulations and experimental results, which validate the presented approach. Concluding remarks are left to Section V.

\section{Visibility MEASUREMENT}

This section presents the principles for obtaining calibrated visibilities with a receiver pair. First, the basics of a regular baseline are presented, and then, the mixed baselines are discussed. The analysis is presented using the MIRAS hardware as an example, but the same principle can be applied to any system which measures normalized correlation.

\section{A. Visibility-Measurement Principle}

Each receiver of the MIRAS array downconverts the signal to intermediate frequency, yielding their in-phase and quadrature components. MIRAS uses 1-bit two-level (1B/2L) correlators to retrieve the correlation between each receiver pair $k, j$ in order to determine the samples of the visibility function of the scene, which is then translated to brightness-temperature image [19].

The complex normalized correlations are obtained by correlating the in-phase and quadrature components with each other and applying the calibration procedure presented in [21] (including corrections for correlator offset and quadrature error). Then, the visibility of $k, j$ pair is obtained through denormalization and residual correction [19], [22]

$$
\begin{aligned}
\hat{V}_{k j} & =V_{k j}-\delta_{k j} V_{k j U} \\
& =\sqrt{T_{\mathrm{sys}_{A k}}^{A} T_{\mathrm{sys}_{A j}}^{A}} M_{k j}-\delta_{k j} \sqrt{T_{\mathrm{sys}_{U k}}^{A} T_{\mathrm{sys}_{U j}}^{A}} M_{k j U}
\end{aligned}
$$

where $V_{k j}$ and $M_{k j}$ are the denormalized and normalized correlations between receivers $k$ and $j$, respectively; $V_{k j U}$ and $M_{k j U}$ are the denormalized and normalized residual correlations between receivers $k$ and $j$, respectively; $\delta_{k j}$ is a coefficient to account for the halved observation time in the case of mixed baselines, for regular baselines $\delta_{k j}=1 ; T_{\mathrm{sys}_{A k}}^{A}$ and $T_{\mathrm{sys}_{A j}}^{A}$ are the system temperatures of receivers $k$ and $j$, respectively, during antenna measurement (subscripted $A$ ) referenced to the antenna input plane (superscripted $A$ ); and $T_{\text {sys }_{U k}}^{A}$ and $T_{\text {sys }_{U j}}^{A}$ are the system temperatures of receivers $k$ and $j$, respectively, during uncorrelated (U)-load measurement referenced to the antenna input plane. The residual correlation, i.e., an offset in the measured correlation, is determined by measuring the U-loads of the receivers, see Fig. 1.

When $k$ and $j$ are LICEF units, the system temperatures are solved as presented in [22], whereas $M_{k j}$ is computed as [26]

$$
M_{k j}=\sin \left(\frac{\pi}{2} Z_{k j}^{i i}\right)+i \sin \left(\frac{\pi}{2} Z_{k j}^{q i}\right)
$$

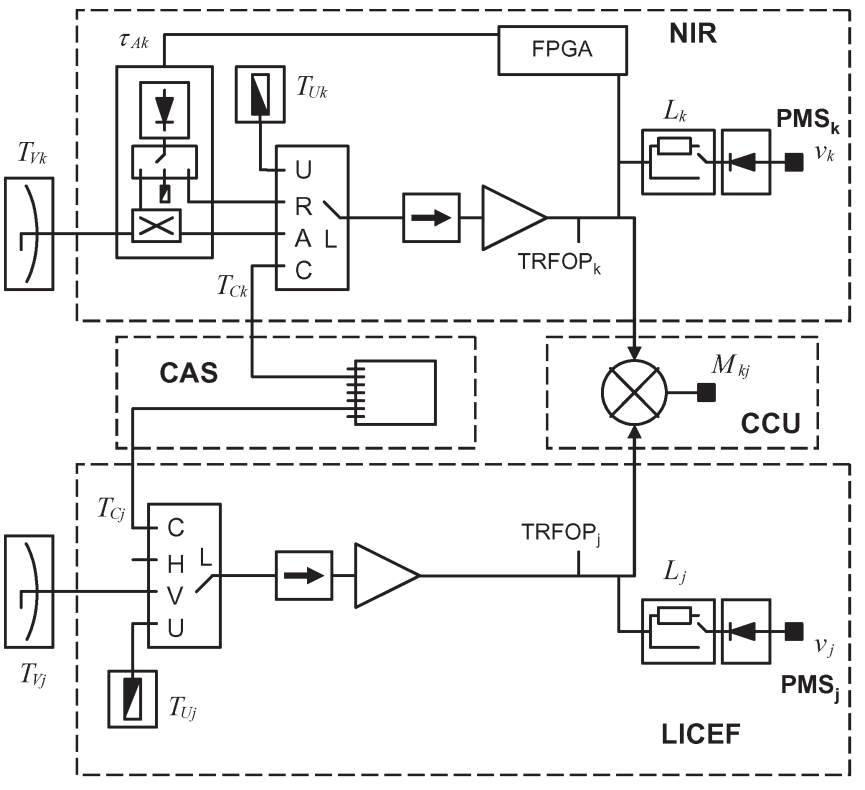

Fig. 1. Schematic diagram of a mixed baseline with one channel of a reference radiometer (NIR) and one regular receiver (LICEF) connected to the CAS, which is a noise-distribution network, and to the central correlator unit (CCU).

where $Z_{k j}^{i i}$ and $Z_{k j}^{q i}$ are the digital correlations, in the range $(-1,1)$, given by the digital correlator $(1 \mathrm{~B} / 2 \mathrm{~L}$ in the case of MIRAS). Superscript $i i$ stands for the correlation between inphase channels, and superscript $q i$ stands for the correlation between quadrature and in-phase channels. However, in the following, no separation is made between the two. The digital correlation is computed from the number of counts $N_{c}$, which is the accumulated number of sign coincidences between the two channels for a given period of time (after which the accumulator resets), as follows:

$$
Z_{k j}=2 \frac{N_{c}}{N_{\max }}-1
$$

where $N_{\max }$ is the maximum number of counts in the given period of time. Note that the fraction $N_{c} / N_{\max }$ is the digital correlation in the range $(0,1)$.

When either $k$ or $j$, or both of them, are channels of NIR units, the denormalization needs special attention due to the noise-injection operation. The baselines which consist of channels of a LICEF unit and a NIR unit are called mixed baselines.

\section{B. Visibility Measurement With Mixed Baselines}

Fig. 1 shows a schematic diagram of a mixed baseline; it is composed of one channel of a NIR unit and one LICEF unit. Subsequently, the formulation of the denormalization of mixed baselines is presented, and then, special attention is paid on the so-called leakage term.

1) Formulation: If one of the receivers of a baseline pair include NIR channels, the integration period is divided into sections according to the state of the noise-injection operation. Fig. 2(a) shows a schematic diagram of the power-detector 


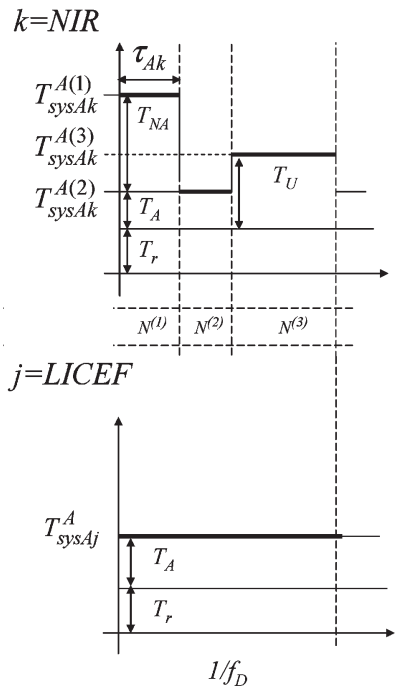

(a)

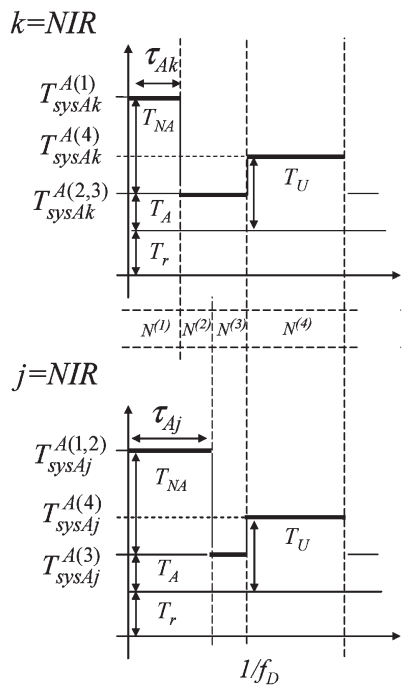

(b)
Fig. 2. (a) Mixed baseline. (b) NIR-NIR baseline. In the case of mixed baselines, Dicke cycles are divided in three steps according to noise injection of the NIR channel, whereas in the case of NIR-NIR baselines, Dicke cycles are divided in four steps according to noise-injection phases of the two NIR channels.

output of the two channels of a mixed baseline: NIR channel goes through the different steps for balancing the output, while the LICEF channel is switched to the antenna for the entire period. During each step, the correlator produces the number of counts $N_{c}^{(p)}$, and therefore, the entire number of counts during the integration time becomes

$$
N_{c}=N_{c}^{(1)}+N_{c}^{(2)}+N_{c}^{(3)} .
$$

On the other hand, the maximum number of counts on each step is as follows: During step 1, when the noise is injected, $N_{1}=1 / 2 N_{\max } \tau$, during step 2 , when no noise is injected, $N_{2}=1 / 2 N_{\max }(1-\tau)$, and during step 3 , when the reference load is measured, $N_{3}=1 / 2 N_{\max }$, based on the noise-injection length $\tau$ as a fraction of the half of the Dicke cycles.

Now, using (3) and (4), the measurement of the digital correlation for a mixed baseline between receivers $k$ and $j$ can be written as

$$
Z_{k j}=\frac{1}{2}\left(\tau_{A k} Z_{k j}^{(1)}+\left(1-\tau_{A k}\right) Z_{k j}^{(2)}+Z_{k j}^{(3)}\right)
$$

where $\tau_{A k}$ is the noise-injection length in NIR-channel $k$ in the antenna measurement mode (see also Figs. 1 and 2) and $Z_{k j}^{(p)}$ is the digital correlation during noise-injection step $p$. Note that step 3 would ideally yield zero correlation.

Using (1), (2), and (5), the relation between the digital correlation during step $p$ and the visibility can be written as

$$
Z_{k j}^{(p)}=\frac{2}{\pi} \sin ^{-1}\left(M_{k j}^{(p)}\right)=\frac{2}{\pi} \sin ^{-1}\left(\frac{1}{\sqrt{T_{\mathrm{sys}_{A k}}^{A(p)} T_{\mathrm{sys}_{A j}}^{A(p)}}} V_{k j}\right) .
$$

Now, using linearization $\sin (x) \approx x, \quad x \ll 1$ (practical limitations for using this approximation are discussed in
Section IV), the total normalized correlation of a mixed baseline can be written as

$$
\begin{gathered}
M_{k j} \approx \frac{1}{2}\left(\tau_{A k} \frac{1}{\sqrt{\left(T_{\mathrm{sys}_{A k}}^{A}+T_{N_{A k}}^{A}\right) T_{\mathrm{sys}_{A j}}^{A}}}\right. \\
+\left(1-\tau_{A k}\right) \frac{1}{\sqrt{T_{\mathrm{sys}_{A k}}^{A} T_{\mathrm{sys}_{A j}}^{A}}} \\
\left.+\frac{S_{L A k}^{U-A}}{\sqrt{L_{A C k}}} \frac{1}{\sqrt{T_{\mathrm{sys}_{U k}}^{U} T_{\mathrm{sys}_{A j}}^{A}}}\right) V_{k j}
\end{gathered}
$$

where $T_{N_{A k}}^{A}$ is the noise-injection temperature of the NIRchannel $k$ referenced to the antenna input plane (solved during the external calibration of the NIR [24]); $S_{L V k}^{U-A}$ is the $S$-parameter describing the isolation between the antenna measurement and U-load measurement (discussed more in Section II-B2); $L_{A C k}$ is the loss of the antenna and its connection of receiver $k$ (solved in the ground calibration of the NIR [24]), the phase of the loss is accounted for in the phase calibration of the baselines; and $T_{\mathrm{sys}_{U k}}^{U}$ is the system noise temperature of receiver $k$ during $\mathrm{U}$-load measurement.

For reference radiometer $k$, the system noise temperature during antenna measurement at the antenna input plane is solved as

$$
T_{\mathrm{sys}_{A k}}^{A}=T_{A_{k}}+T_{\text {rec }_{k}}^{A}
$$

where $T_{A_{k}}$ is the antenna brightness temperature and $T_{\text {rec }_{k}}^{A}$ is the receiver noise temperature referenced to the antenna plane. $T_{A_{k}}$ is solved by the reference radiometer itself, as explained in [24]. $T_{\mathrm{rec}_{k}}^{A}$ is solved at calibration-subsystem (CAS) input plane using the four-point method [22], [32] and transferred to the antenna input plane by applying the losses of the front-end.

2) Leakage Term: The last term in (7) is due to the leakage of the signal to the receiver while the NIR channel is measuring the U-load. The system noise temperature during U-load measurement is retrieved as

$$
\begin{aligned}
T_{\mathrm{sys}_{U k}}^{U} & =T_{U_{k}}+\frac{\left|S_{L A k}^{U-A}\right|^{2}}{L_{A C k}}\left(T_{A_{k}}-T_{U_{k}}\right)+T_{\text {rec }_{k}}^{U} \\
& \approx T_{U_{k}}+T_{\text {rec }_{k}}^{U}
\end{aligned}
$$

where $T_{U_{k}}$ is the U-load noise temperature, which equals to the physical temperature of the receiver, and $T_{\text {reck }_{k}}^{U}$ is the receiver noise temperature at U-load. The approximation can be made, since the isolation parameter is small and its effect on the total system noise is therefore negligible (which, however, is not the case for the visibility).

Now, the isolation parameter $S_{L A k}^{U-A}$ deserves some special attention: It is defined as the difference between $S 21$ parameters measured from $A$ input of the switch to the test output, TRFOP, when the switch is pointed to $U$ and $A$ positions, respectively (see Fig. 1). This way, it describes the leakage of the antenna signal in to the receiver during U-load measurement. The isolation parameter is complex, and thus, this leakage term has also an effect on the phase of the visibility. 
TABLE I

Denormalization Coefficient for all Baselines. In the CASE OF NIR-NIR Baselines, it Is Assumed That $\tau_{A k}>\tau_{A j}$

\begin{tabular}{|c|c|c|c|c|}
\hline$k$ & $j$ & \multicolumn{2}{|r|}{$\Lambda_{k j}$} & $\delta_{k j}$ \\
\hline LICEF & LICEF & \multicolumn{2}{|r|}{1} & 1 \\
\hline NIR & LICEF & $\frac{1}{2}$ & $\left.\left.\tau_{A k} \sqrt{\frac{T_{s y s_{A k}}^{A}}{T_{s y s_{A k}^{A}}^{A}+T_{N_{A k}}^{A}}}+1-\tau_{A k}+\frac{S_{L V k}^{U-A}}{\sqrt{L_{A C k}}} \sqrt{\frac{T_{s y s_{A k}}^{A}}{T_{s y s_{U k}}^{U}}}\right)\right]$ & 2 \\
\hline NIR & NIR & $\frac{1}{2}\left(\tau_{A j} \sqrt{\frac{\left(T_{s y s}^{A} A k\right.}{T_{s}}}\right.$ & $\frac{T_{s y s_{A k}}^{A} T_{s y s_{A j}}^{A}}{\left.+T_{N_{A k}}^{A}\right)\left(T_{s y s_{A j}}^{A}+T_{N_{A j}}^{A}\right)}+\left(\tau_{A k}-\tau_{A j}\right) \sqrt{\frac{T_{s y s_{A k}}^{A}}{T_{s y s_{A k}}^{A}+T_{N_{A k}}^{A}}}+\left(1-\tau_{A k}\right)$ & 2 \\
\hline
\end{tabular}

\section{Visibility Measurement With NIR-NIR Baselines}

If both of the receivers of a baseline pair include noiseinjection channels, the integration period is divided into four sections according to the state of the noise-injection operation in the channels. Fig. 2(b) shows the power-detector output of the two channels. Note that the two channels may represent horizontal and vertical channels in any polarimetric radiometer or two physically separated interferometer channels.

Using the same method as previously discussed, the measurement of the digital correlation for a NIR-NIR baseline between receivers $k$ and $j$ can be written as (if the noise injection is longer in receiver $j$ )

$$
Z_{k j}=\frac{1}{2}\left[\tau_{A k} Z_{k j}^{(1)}+\left(\tau_{A j}-\tau_{A k}\right) Z_{k j}^{(2)}+\left(1-\tau_{A j}\right) Z_{k j}^{(3)}+Z_{k j}^{(4)}\right] .
$$

The last term in the equation can be neglected, since it includes the product of the two isolation terms. As demonstrated in the following sections, only in the case of one isolation factor (i.e., mixed baselines), the impact is low but not negligible. Now, the denormalization equation can be formulated as for the mixed baselines and will be presented in Section III.

\section{Visibility-Measurement Calibration Summary for MIRAS}

Apart from the denormalization, the calibration of the mixed baselines is performed as that of the regular baselines in MIRAS. The calibration is summarized in [20]. As stated before, first, the correlator offset and quadrature error is removed by the self-calibration. The phase calibration is performed by using the front-end phase differences obtained in the ground calibration and the CAS measurements in the orbit. From the denormalized correlations, the residual correlation, or U-correlation, is removed, which is determined by measuring the internal uncorrelated loads of each receiver. Note that, in the correction of the residual correlation, the residual correlation $V_{k j U}$ needs to be multiplied by two in order to account for the halved observation time of the scene visibility, i.e., $\delta_{k j}=2$ in (1). Finally, the antenna patterns (measured on the ground) are accounted for, and second-order corrections are made by the flat target transform [20], [27].

\section{Summary of Denormalization}

This section summarizes the denormalization of all baselines. For the mixed baselines, (7) can be modified to the follow- ing form:

$$
V_{k j}=\Lambda_{k j} \sqrt{T_{\mathrm{sys}_{A k}}^{A} T_{\mathrm{sys}_{A j}}^{A}} M_{k j}
$$

where $M_{k j}$ is obtained over the entire integration time (including all Dicke steps) and in which the denormalization coefficient is defined as

$$
\begin{aligned}
\Lambda_{k j}=\left[\frac { 1 } { 2 } \left(\tau_{A k} \sqrt{\frac{T_{\text {sys }_{A k}}^{A}}{T_{\text {sys }_{A k}}^{A}+T_{N_{A k}}^{A}}}\right.\right. \\
+1-\tau_{A k}+\frac{S_{L A k}^{U-A}}{\sqrt{L_{A C k}}} \sqrt{\left.\left.\frac{T_{\text {sys }_{A k}}^{A}}{T_{\text {sys }_{U k}^{A}}^{A}}\right)\right]^{-1}} .
\end{aligned}
$$

In this way, the effect of the noise injection is isolated to single variable $\Lambda_{k j}$ which can be calculated separately for all baselines. Furthermore, by applying $\Lambda_{k j}$ alone to the measured normalized cross correlations, the normalized correlations of mixed baselines become essentially comparable with normalized correlations of regular baselines, which is useful in debugging and preprocessing of data.

Similarly, the denormalization coefficient can be formulated also for polarimetric zero baselines. Table I summarizes the denormalization coefficient factor for all baselines; in the case of regular baselines, the value is naturally one. Additionally, Table I summarizes also the factor for the residual correlation correction.

The third and fourth Stokes parameters, according to the definition presented, e.g., in [28] and [29], are directly given by visibility $\hat{V}_{k j}$ when $k=H$ and $j=V$

$$
\begin{aligned}
& T_{3_{k j}}=2 \Re e\left\{\hat{V}_{k j}\right\} \\
& T_{4_{k j}}=2 \Im m\left\{\hat{V}_{k j}\right\}
\end{aligned}
$$

where receivers $k$ and $j$ can be those of either LICEF or NIR units. Note that this is equivalent to (5) in [1] but now applied to any type of receiver.

\section{EFFECTS OF LINEARIZATION AND ISOLATION}

This section investigates the effects of the linearization of (6) and the isolation parameter $S_{L A k}^{U-A}$ on the measurement of mixed baselines. Experimental results on the denormalization of the mixed baselines are presented. The mixed baselines are compared to the regular baselines (obtained so that NIR units 


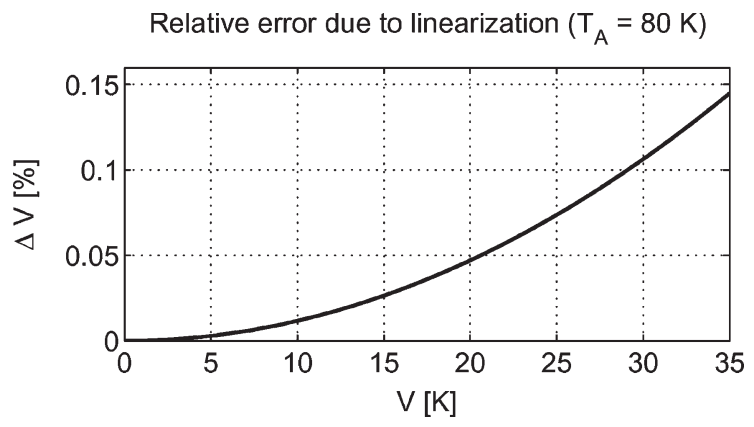

Fig. 3. Simulated relative error of the visibility due to the linearization as a function of the visibility.

are in total power-measurement mode), and the denormalization equation, with the leakage term, is validated.

\section{A. Linearization of Correlation Transform}

The effect of the linearization approximation of (6) as presented in Section II-B can be evaluated by solving (6) accurately numerically.

Fig. 3 shows a plot of the relative error between linearized and accurate solution of (6). In order to obtain a worst case scenario, a low value for antenna temperature was used: $T_{A}=$ $T_{H_{k}}=T_{H_{j}}=80 \mathrm{~K}$, which could theoretically be measured from ocean surface. Other used values were as follows: $T_{\text {rec }_{k}}=$ $260 \mathrm{~K} ; T_{\text {rec }_{j}}=170 \mathrm{~K} ; T_{N A_{k}}=550 \mathrm{~K} ; T_{p h}=21{ }^{\circ} \mathrm{C}$ (the same physical temperature was applied to the entire front-end); the isolation was assumed infinite; and the normalized correlation $M_{k j}$ ranged from 0 to 0.06 (or $600 \mathrm{cu}$ as $1 \mathrm{cu}=10^{-4}$ ).

Simulations (e.g., with SMOS end-to-end performance simulator [30]) show that, for natural targets, the amplitude of $V_{k j}$ is lower than $30 \mathrm{~K}$. Therefore, the linearization error is lower than $0.1 \%$ and can be neglected. However, for an artificial point source, the visibility amplitude can be as large as $1000 \mathrm{~K}$ (see Fig. 6). In this case, (6) cannot be linearized and is solved by numerical means.

\section{B. Effect of Isolation Parameter}

The effect of the isolation parameter on denormalized correlations was analyzed with different values of isolation parameter. The same values as in the previous section were used for the radiometer, but the normalized correlation was set to $600 \mathrm{cu}$ (or 0.06) and the antenna temperature was set to $250 \mathrm{~K}$, since it now yields the worst case. Fig. 4 shows the resulting error. In the case of LICEF, the magnitude is about $4 \%$ (see Table II), and therefore, it is clear that this effect needs to be accounted for.

1) Measured S-Parameters for Isolation: In the groundcalibration campaign of the LICEF units, extensive $S$-parameter measurements of the front-ends of each LICEF unit were carried out by the manufacturer Mier Communicaciones, Spain. As a consequence, the isolation parameters can also be retrieved from these measurements at three temperature points (namely, $5{ }^{\circ} \mathrm{C}, 21{ }^{\circ} \mathrm{C}$, and $45{ }^{\circ} \mathrm{C}$ ). Table II shows the measured isolation parameters $S_{L A k}^{U-A}$ for each NIR

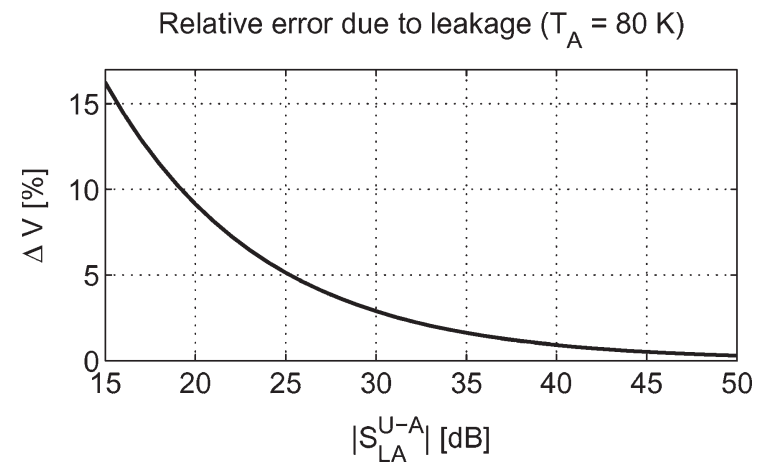

Fig. 4. Simulated relative error of the visibility due to leakage as a function of the isolation parameter.

TABLE II

ISOLATION PARAMETERS AT NOMINAL PHySICAL TEMPERATURE

\begin{tabular}{c||c|c|c|c|c|c}
\hline \hline \multicolumn{1}{c||}{} & \multicolumn{2}{c|}{ NIR-1 } & \multicolumn{2}{c|}{ NIR-2 } & \multicolumn{2}{c}{ NIR-3 } \\
& $V$ & $H$ & $V$ & $H$ & $V$ & $H$ \\
\hline Amplitude [dB] & -27.7 & -28.0 & -27.8 & -27.7 & -27.8 & -27.8 \\
\hline Phase [deg] & 103.2 & 103.5 & 102.7 & 102.7 & 103.3 & 103.3 \\
\hline \hline
\end{tabular}

channel at around the nominal physical temperature $\left(21^{\circ} \mathrm{C}\right)$. The measurements were carried out with a calibrated vector network analyzer between $A$ and TRFOP ports of the LICEF units (see Fig. 1) as required by the definition of the isolation parameter (see Section II-B2).

The results show that the isolation is very similar in each front end with respect to both amplitude and phase. It is also noted that the results are very similar at each measured temperature. The magnitude of the isolation is regarded as reasonable for this kind of single-pole four-throw switch, which have been optimized for low loss to improve radiometric sensitivity.

2) Point-Source Measurements: The point-source measurements were carried out as part of the image validation test of MIRAS, which took place in the Maxwell EMC chamber of the test facility of ESA's European Space Research and Technology Center.

Fig. 5 shows a schematic diagram of the setup of the pointsource measurements. The setup follows the principles laid out in [31]. MIRAS was deployed on the floor so that the antennas were pointing upward to the ceiling. The point source, i.e., an antenna connected to a noise source, was located in the ceiling pointing downward to MIRAS. The antenna was aligned with the center of MIRAS. The ceiling, walls, and floor of the chamber were covered with pyramid-shaped absorbers.

Fig. 6 shows the results obtained in the point-source measurement between $V$ channel of NIR-1 and $V$ channels of the LICEF units. The top plots show the result when the isolation parameter is accounted for, and the bottom plots show the result when the isolation parameter is omitted.

The results are retrieved so that the denormalized value obtained in the total power mode of NIR (LICEF-LA) is compared to the one obtained in the noise-injection mode (NIR-A). In the top left plot, these measurements of each baseline, including $V$ channel of NIR-1, coincide with mean accuracy of $0.35 \%$ (top right plot). When the isolation parameter is omitted, the 

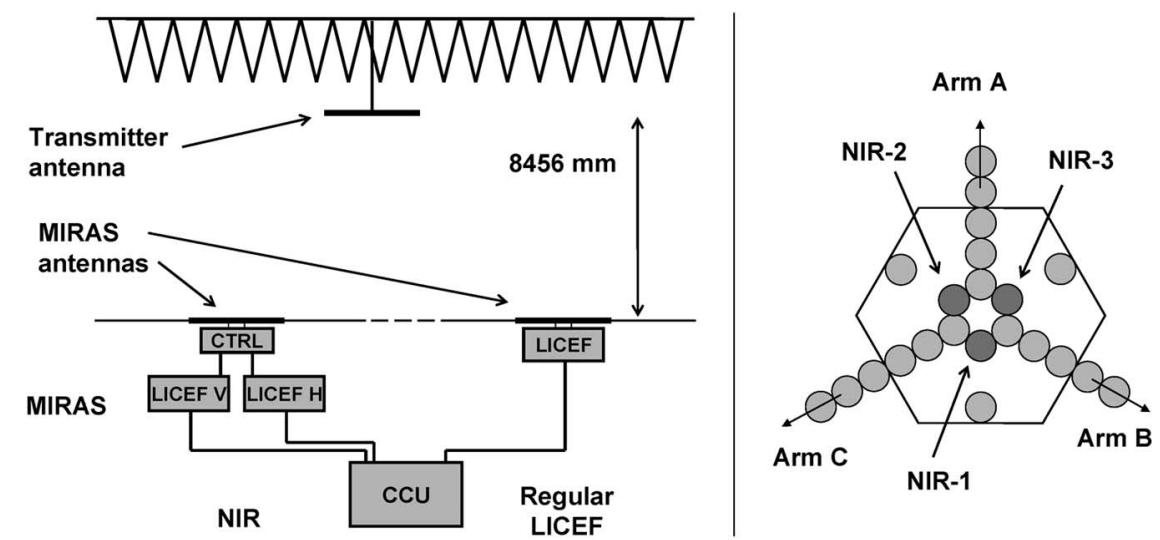

Fig. 5. Schematic diagram of the setup for the point-source measurements (on the left-hand side) and a layout diagram of the NIR units in the MIRAS array (on the right-hand side).
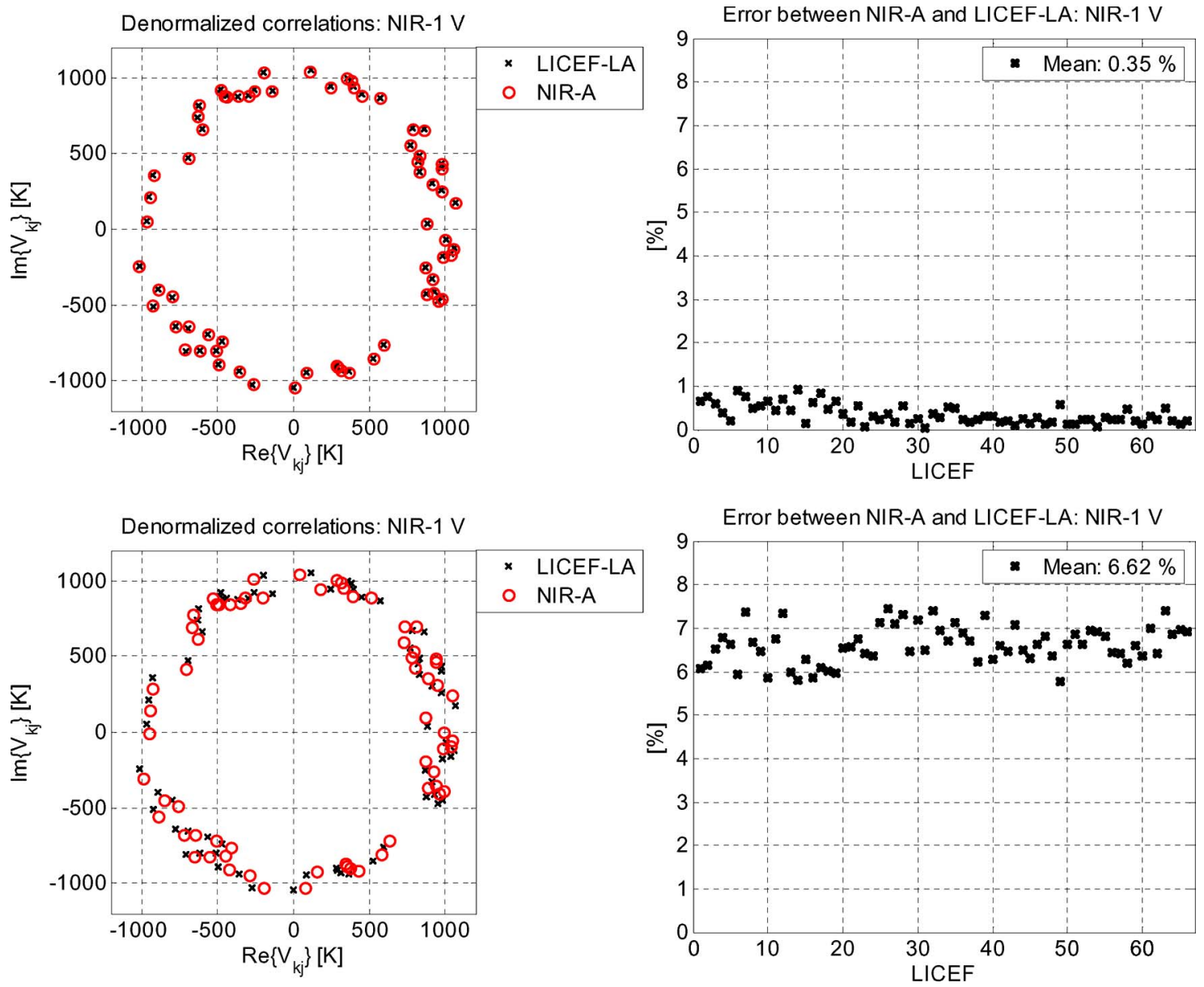

Fig. 6. On the top left, the denormalization results of the points-source measurements for the $V$ channel of NIR-1 so that the NIR is in total power (LICEF-LA) and noise-injection modes (NIR-A). On the top right, the relative difference between total power and noise-injection mode results. On the bottom left and right, the respective plots obtained so that the isolation parameter is not accounted for.

measurements clearly do not coincide (bottom left plot), and the mean error is over $6 \%$ (bottom right plot). The results obtained with the other NIR channels are similar, and the total mean difference between the total power measurement and the noiseinjection measurement including all mixed baselines of MIRAS is $0.50 \%$.

Note that, as the magnitude of the point source is high, the linearization approximation of the correlation transform of
(6) cannot be applied, and the transform has to be calculated accurately by numerical means.

\section{CONCLUSION}

The denormalization of normalized correlations measured in the presence of switching and noise injection was analyzed, and a unifying formulation for the denormalization of all types of 
correlations - regular, mixed, and polarimetric zero baselineswas introduced. The analysis was applied to mixed baselines, those baselines involving the reference NIRs, of the MIRAS instrument of the SMOS mission.

Additionally, the effect of the linearization of the transform from digital to analog correlation was evaluated for the case of $1 \mathrm{~B} / 2 \mathrm{~L}$ correlator. It was concluded that, for the normal operation of the MIRAS instrument, the linearization can be made due to the low magnitude of visibility values. This simplifies the processing significantly. However, in the calibration tests such as the measurements performed in the anechoic chamber, the equations cannot be linearized and have to be solved numerically.

It was also shown that the finite isolation in the front-end switch affects the measurement of the visibility of the mixed baselines. Furthermore, the isolation parameter of the switch can be determined from the $S$-parameters of the front-end and then applied as a correction, which was demonstrated using point-source measurements.

The presented methods can be applied to any radiometer system, which has noise injection and/or switching in the measurement chain and utilizes measurement of normalized correlations (e.g., imaging synthetic aperture or polarimetric radiometers).

\section{ACKNOWLEDGMENT}

The authors would like to thank Mier Communicaciones, Spain, for providing the data of the LICEF test campaign. They would also like to thank several people from EADS-CASA Espacio, UPC, and ESA for contributing to the MIRAS Image Validation Tests and for acquiring the data.

\section{REFERENCES}

[1] J. R. Piepmeier and A. J. Gasiewski, "Digital correlation microwave polarimetry: Analysis and demonstration," IEEE Trans. Geosci. Remote Sens., vol. 39, no. 11, pp. 2392-2410, Nov. 2001.

[2] D. M. Le Vine, "Synthetic aperture radiometer systems," IEEE Trans. Microw. Theory Tech., vol. 47, no. 12, pp. 2228-2236, Dec. 1999.

[3] D. M. Le Vine, A. J. Griffis, C. T. Swift, and T. J. Jackson, "ESTAR: A synthetic aperture microwave radiometer for remote sensing applications," Proc. IEEE, vol. 82, no. 12, pp. 1787-1801, Dec. 1994.

[4] J. Kainulainen, K. Rautiainen, S. Tauriainen, T. Auer, J. Kettunen, and M. Hallikainen, "First 2-D interferometric radiometer imaging of the Earth from an aircraft," IEEE Geosci. Remote Sens. Lett., vol. 4, no. 2, pp. 241-245, Apr. 2007

[5] S. Ribo, M. Martin-Neira, I. Cabeza, S. Tauriainen, and N. Duffo, "MIRAS airborne demonstrator," in Proc. IEEE MicroRad, San Juan, PR, Feb. 28-Mar. 3, 2006, pp. 45-49.

[6] A. B. Tanner et al., "Initial results of the geostationary synthetic thinned array radiometer (GeoSTAR) demonstrator instrument," IEEE Trans. Geosci. Remote Sens., vol. 45, no. 7, pp. 1947-1957, Jul. 2007.

[7] A. B. Tanner and C. T. Swift, "Calibration of a synthetic aperture radiometer," IEEE Trans. Geosci. Remote Sens., vol. 31, no. 1, pp. 257-267, Jan. 1993.

[8] F. Torres, A. Camps, J. Bará, I. Corbella, and R. Ferrero, "On-board phase and modulus calibration of large aperture synthesis radiometers: Study applied to MIRAS," IEEE Trans. Geosci. Remote Sens., vol. 34, no. 4, pp. 1000-1009, Jul. 1996.

[9] M. Martín-Neira and J. M. Goutoule, "A two-dimensional aperturesynthesis radiometer for soil moisture and ocean salinity observations," ESA Bull., no. 92, pp. 95-104, Nov. 1997.

[10] K. D. McMullan, M. A. Brown, M. Martín-Neira, W. Rits, S. Ekholm, J. Marti, and J. Lemanczyk, "SMOS: The payload," IEEE Trans. Geosci. Remote Sens., vol. 46, no. 3, pp. 594-605, Mar. 2008.
[11] M. Martin-Neira et al., "The SMOS mission," ESA Bull., no. 111, pp. 125-131, Aug. 2002

[12] H. Barré, B. Duesmann, and Y. Kerr, "SMOS: The mission and the system," IEEE Trans. Geosci. Remote Sens., vol. 46, no. 3, pp. 587-593, Mar. 2008.

[13] R. W. Newton and J. W. Rouse, Jr., "Microwave radiometer measurements of soil moisture content," IEEE Trans. Antennas Propag., vol. AP-28, no. 5, pp. 680-686, Sep. 1980.

[14] Y. H. Kerr, P. Waldteufel, J.-P. Wigneron, J. Martinuzzi, J. Font, and M. Berger, "Soil moisture retrieval from space: The soil moisture and ocean salinity (SMOS) mission," IEEE Trans. Geosci. Remote Sens., vol. 39, no. 8, pp. 1729-1735, Aug. 2001.

[15] H.-J. C. Blume, B. M. Kendall, and J. C. Fedors, "Measurement of ocean temperature and salinity via microwave radiometry," Boundary-Layer Meteorol., vol. 13, no. 1-4, pp. 295-308, Jan. 1978.

[16] J. Font, G. S. E. Lagerloef, D. M. Le Vine, A. Camps, and O.-Z. Zanife, "The determination of surface salinity with the European SMOS space mission," IEEE Trans. Geosci. Remote Sens., vol. 42, no. 10, pp. 21962205, Oct. 2004.

[17] C. Ruf, C. T. Swift, A. B. Tanner, and D. M. Le Vine, "Interferometric synthetic aperture microwave radiometry for the remote sensing of the Earth," IEEE Trans. Geosci. Remote Sens., vol. 26, no. 5, pp. 597-611, Sep. 1988.

[18] A. Camps, "Application of interferometric radiometry to Earth observation," Ph.D. dissertation, Universitat Politecnica de Catalunya, Barcelona, Spain, 1996.

[19] I. Corbella, N. Duffo, M. Vall-llossera, A. Camps, and F. Torres, "The visibility function in interferometric aperture synthesis radiometry," IEEE Trans. Geosci. Remote Sens., vol. 42, no. 8, pp. 1677-1682, Aug. 2004.

[20] M. A. Brown, F. Torres, I. Corbella, and A. Colliander, "SMOS calibration," IEEE Trans. Geosci. Remote Sens., vol. 46, no. 3, pp. 646-658, Mar. 2008.

[21] I. Corbella, F. Torres, A. Camps, A. Colliander, M. Martin-Neira, S. Ribo, K. Rautiainen, N. Duffo, and M. Vall-llossera, "MIRAS end-toend calibration: Application to SMOS L1 processor," IEEE Trans. Geosci. Remote Sens., vol. 43, no. 5, pp. 1126-1134, May 2005.

[22] F. Torres, I. Corbella, A. Camps, N. Duffo, M. Vall-llosera, S. Beraza, C. Gutirrez, and M. Martin-Neira, "Denormalization of visibilities for in-orbit calibration of interferometric radiometers," IEEE Trans. Geosci. Remote Sens., vol. 44, no. 10, pp. 2679-2686, Oct. 2006.

[23] A. Colliander, S. Tauriainen, T. Auer, J. Kainulainen, J. Uusitalo, M. Toikka, and M. T. Hallikainen, "MIRAS reference radiometer: A fully polarimetric noise injection radiometer," IEEE Trans. Geosci. Remote Sens., vol. 43, no. 5, pp. 1135-1143, May 2005.

[24] A. Colliander, L. Ruokokoski, J. Suomela, K. Veijola, J. Kettunen, V. Kangas, M. Levander, H. Greus, M. T. Hallikainen, and J. Lahtinen, "Development and calibration of SMOS reference radiometer," IEEE Trans. Geosci. Remote Sens., vol. 45, no. 7, pp. 1967-1977, Jul. 2007.

[25] J. Lemmetyinen, J. Uusitalo, K. Rautiainen, J. Kainulainen, N. Fabritius, M. Levander, V. Kangas, H. Greus, J. Pihlflyckt, A. Kontu, S. Kemppainen, M. T. Hallikainen, and J. Lahtinen, "SMOS calibration subsystem," IEEE Trans. Geosci. Remote Sens., vol. 45, no. 11, pp. 36913700 , Nov. 2007

[26] J. B. Hagen and D. T. Farley, "Digital-correlation techniques in radio science," Radio Sci., vol. 8, no. 8/9, pp. 775-784, Aug./Sep. 1973.

[27] M. Martín-Neira, M. Suess, J. Kainulainen, and F. Martín-Porqueras, "The flat target transformation," IEEE Trans. Geosci. Remote Sens., vol. 46 , no. 3, pp. 613-620, Mar. 2008.

[28] A. Ishimaru, Wave Propagation and Scattering in Random Media, Volume 1, Single Scattering and Transport Theory. New York: Academic, 1978, $250 \mathrm{pp}$.

[29] J. R. Piepmeier, D. G. Long, and E. G. Njoku, "Stokes antenna temperatures," IEEE Trans. Geosci. Remote Sens., vol. 46, no. 2, pp. 516-527, Feb. 2008.

[30] A. Camps, I. Corbella, M. Vall-llosera, N. Duffo, F. Marcos, F. Martinez-Fadrique, and M. Greiner, "The SMOS end-to-end performance simulator: Description and scientific applications," in Proc. IEEE IGARSS, Toulouse, France, Jul. 2003, pp. 13-15.

[31] I. Corbella, F. Torres, S. Blanch, S. Ribo, S. Beraza, N. Duffo, A. Camps, M. Vall-llossera, and M. Martin-Neira, "Inter-element phase calibration in interferometric radiometers," in Proc. IEEE IGARSS, Denver, CO, Jul. 2006, pp. 3976-3979.

[32] P. Piironen, "PMS offset determination using an IF attenuator," ESA/ESTEC, Noordwijk, The Netherlands, Jun. 2002. Technical Note $14629 / 00 / \mathrm{NL} / \mathrm{SF}$. 


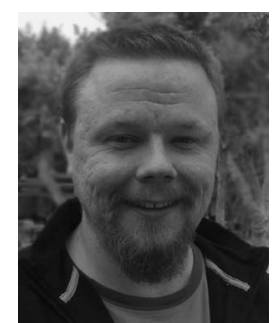

Andreas Colliander (S'04-A'06-M'07-SM'08) was born in Imatra, Finland, in 1976. He received the M.Sc. (Tech.), Lic.Sc. (Tech.), and D.Sc. (Tech.) degrees from the Helsinki University of Technology (TKK), Espoo, Finland, in 2002, 2005, and 2007, respectively.

From 2001 to 2007, he was with the Laboratory of Space Technology, TKK, where he was a Research Scientist, and was also a Project Manager related to the Noise Injection Radiometers in the European Space Agency's (ESA's) SMOS Payload Module Project. From 2007 to 2008, he was a Postdoctoral Research Fellow with the European Space Research and Technology Centre, ESA, Noordwijk, The Netherlands. He is currently a Scientist with the Jet Propulsion Laboratory, California Institute of Technology, Pasadena, where he is a Member of the Science Algorithm Team for the Soil Moisture Active and Passive Mission. He has authored or coauthored over 30 scientific publications on microwave remote sensing. His research has focused on microwave radiometry and theoretical simulation of rough-surface backscattering.

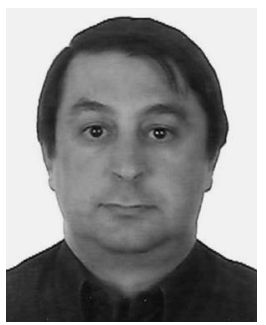

Francesc Torres (S'88-M'96-SM'06) was born in Ibiza, Spain, in 1962. He received the Ingeniero and Doctor Ingeniero degrees in telecommunication engineering from the Universitat Politècnica de Catalunya (UPC), Barcelona, Spain, in 1988 and 1992 , respectively.

From 1988 to 1989 , he was a Research Assistant with the RF System Division, European Space Agency, Holland, where he was devoted to microwave-device testing and characterization. From 1989 to 1996, he was an Assistant Professor of microwave circuits and systems with the Signal Theory and Communications Department, Remote Sensing Laboratory, UPC, where he has been an Associate Professor since 1996. Since 1995, he has been participating in a number of projects related to the SMOS mission by the European Space Agency. From 2005 to 2006, he held a sabbatical stage with the Microwave Systems Section, Jet Propulsion Laboratory, Pasadena, CA, where he was involved in the GeoSTAR pilot project, which is a passive-microwave interferometric geosounder. He is currently coleading the Barcelona SMOS Expert Centre on Radiometric Calibration and Ocean Salinity, Barcelona, Spain.

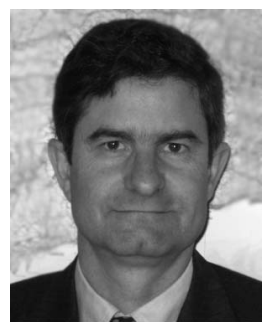

Ignasi Corbella (M'99-SM'08) received the Engineer degree in telecommunication engineering and the Dr.Eng. degree from the Universitat Politècnica de Catalunya (UPC), Barcelona, Spain, in 1977 and 1983, respectively.

In 1976, he was a Research Assistant with the Microwave Laboratory, School of Telecommunication Engineering, UPC, where he worked on passive-microwave integrated-circuit design and characterization. In 1979, he was with ThomsonCSF, Paris, France, where he worked on microwaveoscillators design. In 1982, he was an Assistant Professor, in 1986, an Associate Professor, and in 1993, a Full Professor with UPC, where he is currently teaching basic microwaves and antennas at undergraduate level and graduate courses on nonlinear microwave circuits. Since 1993, he has been actively participating as a Researcher with the European Space Agency (ESA) SMOS mission in the frame of several contracts, directly with ESA, or with the payload prime contractor EADS-Casa Espacio. His expertise includes, among others, fundamentals of interferometric aperture synthesis radiometry, imagereconstruction algorithms, and onboard calibration, hardware specification, and payload characterization. From 1993 to 1997, he was an Academic Director with the School of Telecommunications Engineering. From 1998 to 1999, he was with the NOAA/Environmental Technology Laboratory, as a Guest Researcher developing methods for total-power radiometer calibration and data analysis. From 1999 to 2007, he was the Scientific Coordinator of a Dictionary of Telecommunication terms in Catalan language, with more than 4000 entries, published in March 2007. From 2001 to 2003, he was a Director with the Department of Signal Theory and Communications, UPC. Since 2004, he has been a Member of the SMOS Science Advisory Group, and since 2007, he has been a Member of the SMOS Barcelona Expert Centre.

Dr. Corbella was the General Chairman of the 2007 International Geoscience and Remote Sensing Symposium (IGARSS'07), Barcelona, from July 22-27, 2007. 